\title{
HEMATOMA POSTRAUMÁTICO EN RIÑÓN PATOLÓGICO
}

\author{
M. ALLUÉ LÓPEZ, D. PASCUAL REGUEIRO, A. GARCÍA DE JALÓN MARTÍNEZ, \\ P. SERRANO FRAGO, P. GIL MARTÍNEZ, L.A. RIOJA SANZ
}

Servicio de Urología. Hospital Universitario “Miguel Servet”. Zaragoza.

Actas Urol Esp. 27 (1): 65-67, 2003

\section{RESUMEN}

"HEMATOMA POSTRAUMÁTICO EN RIÑÓN PATOLÓGICO”

Presentamos un caso de hematoma postraumático en un riñón tumoral.

El diagnóstico del hematoma fue realizado mediante escáner abdominal pero no así el tipo tumoral.

Fue necesario la revisión quirúrgica y posterior estudio patológico para demostrar un angiomiolipoma como causante del hematoma renal.

PALABRAS CLAVE: Angiomiolipoma renal. Traumatismo renal.

\section{ABSTRACT \\ "POSTRAUMATIC HEMATOMA IN PATHOLOGICAL KIDNEY"}

We want to present a case of postraumatic hematoma in a tumoral kidney.

The diagnosis of the hematoma was made by abdominal scanner but not the tumor type.

It was necessary the chirurgycal review and patological study to demostrate an angiomyolipoma as the cause of the kidney hematoma.

KEY WORDS: Kidney angiomyolipoma. Traumatic kidney.

$\mathrm{L}$ os traumatismos renales pueden manifestar/se de distintas maneras según las clasificaciones ampliamente conocidas; una de sus formas más leves es el hematoma perirrenal y/o subcapsular cuyo tratamiento estandarizado es la vigilancia expectante, mientras no haya alteraciones hemodinámicas o descenso brusco del hematocrito. En dicha patología es fundamental el diagnóstico por la imagen siendo la ECO y especialmente el TAC las pruebas que más rendimiento ofrecen, permitiendo a su vez un seguimiento y control evolutivo.

Además de los traumatismos el sangrado renal en forma de hematoma perirrenal o subcapsular puede estar motivado por la existencia de una patología previa, como son las neoplasias renales. En este punto es el angiomiolipoma la tumoración renal que más frecuentemente se asocia a sangrado, siendo además el responsable en la mayor parte de las ocasiones del conocido sindrome de Wünderlich.
A continuación presentamos el caso de un hematoma renal postraumático sobre un riñón con patología tumoral de base, que requirió la intervención quirúrgica para su filiación definitiva.

\section{CASO CLÍNICO}

Paciente mujer de 65 años que es atendida en el Servicio de urgencias de traumatología por presentar un extenso hematoma afectando al muslo izquierdo, como consecuencia de un accidente de tráfico (la paciente fue atropellada mientras cruzaba la calle).

$\mathrm{Al}$ ingreso se descarta patología ósea y se decide tratamiento conservador y reposo. Doce días después se descubre en un control analítico un descenso importante del hematocrito que es achacado al hematoma del muslo, no obstante la paciente comienza a aquejar molestias en el flanco izquierdo, que una vez interrogada dice presentar desde el momento del atropello. 
Se solicita ecografía abdominal para descartar rotura de bazo. Se halla voluminoso hematoma subcapsular renal izquierdo de $15 \times 9 \times 7 \mathrm{~cm}$. Con este diagnóstico se nos consulta para la valoración de la paciente, la cual se encuentra hemodinámicamente estable, sin presentar hematuria y con un leve dolor en el flanco izquierdo. Se solicita TAC que demuestra los mismos hallazgos que la ecografía aunque da la sensación de que dicho hematoma presenta una muy fina y heterogénea captación de contraste en su porción más interna, así como un angiomiolipoma de $35 \mathrm{~mm}$ en polo inferior del riñón derecho (Fig. 1).

Se decide en un principio conducta expectante dada la situación estable de la paciente. Diez días después se repite el TAC que demuestra la persistencia del hematoma subcapsular con leves modi-
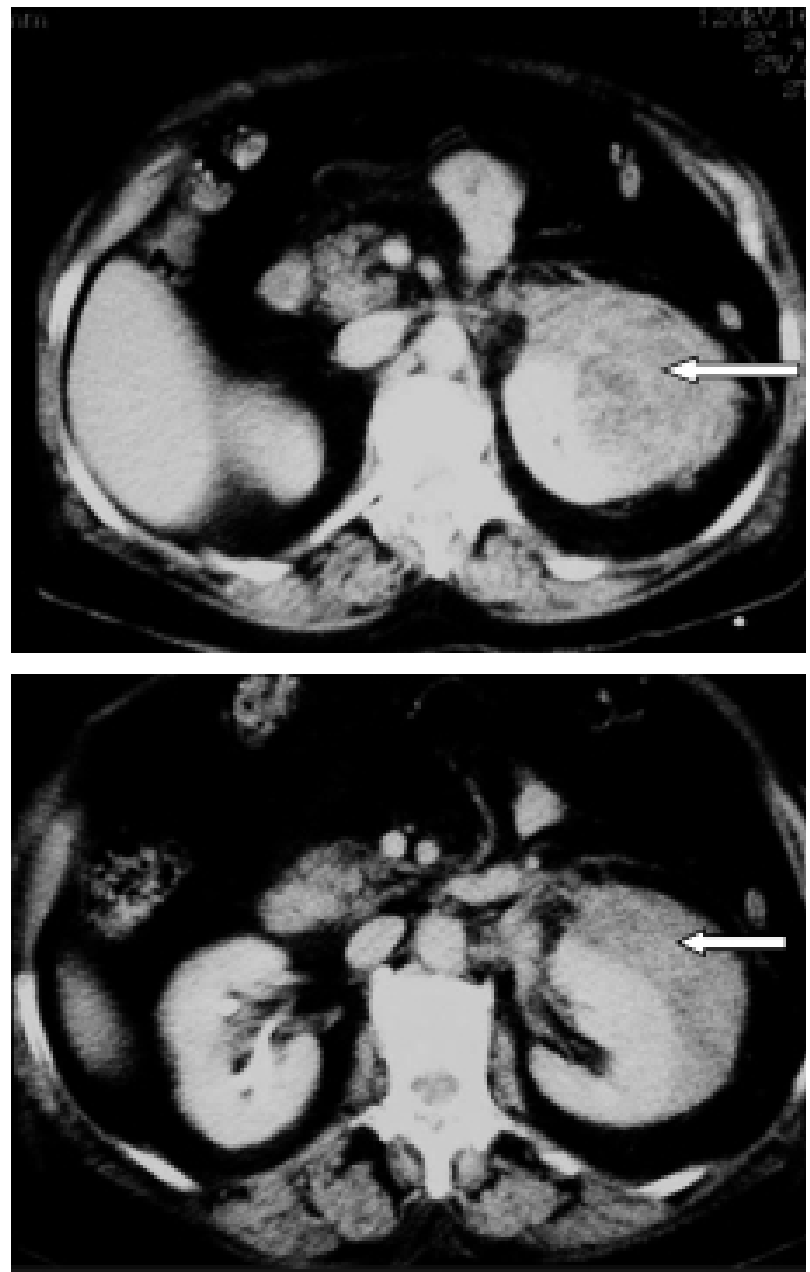

FIGURA 1. Imagen de TAC en la que se aprecia el gran hematoma subcapsular renal (flechas blancas). En el corte superior se pone de manifiesto una zona con discreta captación heterogénea de contraste. ficaciones respecto al control previo pero en este caso se evidencia en la zona interna del hematoma una lesión sólida nodular en el polo superior renal de 42 x $40 \mathrm{~mm}$ con claro realce post-contraste, lo que confirma que se trata de una lesión tumoral. En dichas imágenes no se evidencia claramente densidad grasa, por lo que no puede establecerse el diagnóstico diferencial entre carcinoma de células renales y angiomiolipoma (Fig. 2).
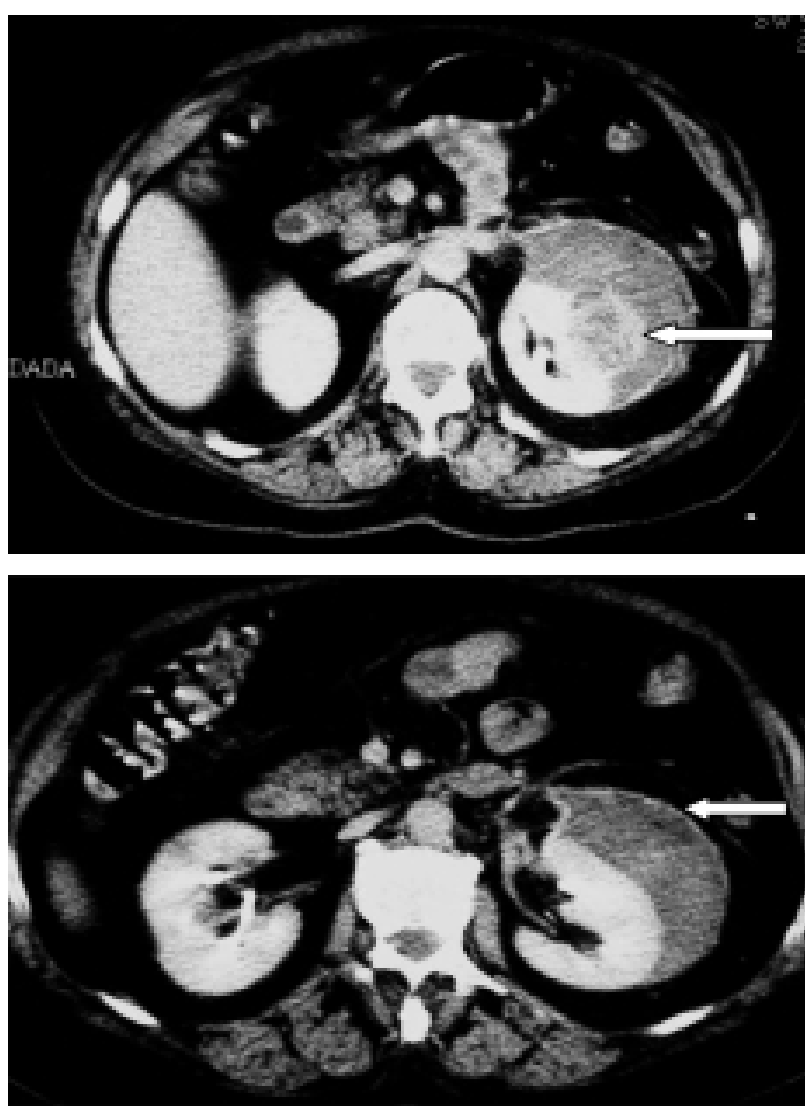

FIGURA 2. Imagen de control del hematoma diez dias después. Apenas existe modificación en su tamaño, pero si que se evidencia, en el corte superior, la presencia de una lesión sólida con captación heterogénea de contraste flecha blanca), aunque no se aprecia densidad grasa en su interior. En el corte inferior se puede apreciar la decapsulación renal.

La situación de la paciente es buena con estabilización y elevación del hematocrito sin requerir transfusión. Se decide la revisión quirúrgica que se lleva a cabo 6 días después. En dicha intervención se realiza una lumbotomía izquierda con resección de la $12^{\underline{a}}$ costilla. Se evidencia un gran hematoma decapsulando gran parte de la cara anterior y del polo superior renal. 
En dicho polo superior se observa una tumoración de aspecto heterogéneo de un tamaño aproximado de $42 \times 40 \mathrm{~mm}$ y que al corte sugiere un carcinoma de células renales. Dada la decorticación importante de riñón, el hematoma de gran tamaño y las dimensiones tumorales se decide llevar a cabo nefrectomía izquierda. En el mismo acto, el Servicio de Traumatología procede al drenaje del hematoma del muslo izquierdo.

El post-operatorio transcurre sin incidencias siendo dada de alta a los 10 días tras la intervención.

$\mathrm{El}$ análisis anatomopatológico de la pieza puso de manifiesto la presencia de un angiomiolipoma de $45 \mathrm{~mm}$, encontrándose el resto del riñón sin alteraciones. La paciente actualmente se encuentra bien con una función renal normal (creatinina plasmática $1 \mathrm{mg} / \mathrm{dl}$ ).

\section{DISCUSIÓN}

Nos encontramos ante un caso de un traumatismo renal sobre un riñón patológico que ha favorecido su sangrado. La falta de manifestaciones clínicas y la existencia de una patología traumatológica más evidente, determinaron que dicho traumatismo pasara en un principio desapercibido. Con respecto a este punto podríamos defender la necesidad de una exploración más exhaustiva después de un traumatismo violento como resulta un accidente de tráfico.

La ecografía demuestra su gran eficacia en el diagnóstico de lesiones renales añadido a la sencillez y rapidez de su realización. No obstante, la existencia de un gran hematoma subcapsular produjo el enmascaramiento de la patología tumoral de base, que de otro modo se hubiera puesto de manifiesto en la mayor parte de las ocasiones.

La prueba de imagen más definitoria en los traumatismos renales es sin duda el TAC, que nos permite conocer el tamaño, grado y características de las lesiones, permitiendo descartar situaciones de gran urgencia y gravedad como son la rotura del parénquima renal y de los vasos arteriales y/o venosos.

Dicha prueba tiene también alta resolución a la hora del diagnóstico de los angiomiolipomas mediante la detección intratumoral de densidad grasa, como así lo demuestra con la evidencia de un angiomiolipoma en el riñón derecho de nuestra paciente.

Sin embargo la resolución del TAC no fue suficiente para permitirnos el diagnóstico tumoral inicial ni el de angiomiolipoma posteriormente, posi- blemente por el artefacto que supone la presencia del hematoma subcapsular de grandes dimensiones que afectaba a dicho riñón.

El tratamiento de los traumatismos renales de bajo grado, siempre que no haya repercusión clínico-hemodinámica, ni descenso grave del hematocrito, es la conducta expectante, con reposo en cama y con controles seriados de hematocrito, constantes hemodinámicas y repetición de pruebas de imagen para la valoración de la evolución del hematoma. En nuestro caso y a pesar de la mejoría clínica de la paciente, el descubrimiento en el TAC de control de una masa sólida como causante del sangrado, obligó a su revisión quirúrgica.

La lumbotomía exploradora no nos permitió un diagnóstico claro del tipo de lesión tumoral.

Su localización ocupando la zona del polo superior y mesorrenal, su asociación al gran hematoma y la decapsulación importante de gran parte del riñón, nos llevaron a decidirnos por una nefrectomía total como tratamiento más adecuado. No obstante, somos conscientes que en otra situación podría haberse intentado una cirugía conservadora, previa confirmación intraoperatoria de la benignidad de la tumoración.

\section{REFERENCIAS}

1. MNIF A, BEN MOUALLI S, LOUSSAIEF H, AYED M.: Isolated, spontaneous hematoma of the kidney. Report of two cases. Hospital Charles Nicolle de Tunis. Service d'Urologie Tunis Med 2001 mar; 79 (3): 195-198.

2. GOMES ALMEIDA F, REIS J, RIBEIRO AM.: Traumatic rupture of angiomyolipoma: a case report. Servicio de Radiologia. Hospital General de Santo Porto, Portugal. Arch Esp Urol 2000 apr; 53 (3): 286-289

3. WILSHIRE P, ANDRE M, GROS N, SAMAMA D, CROZIER F, VIDAL V, PASCAL T, BARTOLI J.: Primitive neuroectodermal tumor of the kidney manifested as a spontaneous hematoma. Service central de radiologie, Hospital de la Timone, 264, rue Sanint Pierre, 13385 Marseille, France. J Radiol 2000 mar; 81 (3): 237-240.

4. WOLFENSBERGER UE, KUBIK-HUCH RA, SULSER T, PFAMMATTER T, MARINCEK B.: Acute rupture of a solitary angiomyolipoma in the upper pol of the right kidney with extensive perineal hematoma. Institut fur diagnostische Radiologie, Universitatsspital Zurich. Schweiz Rundsch Med Prax 1998 nov 4; 87 (45): 1.509-1.511.

5. FERNÁNDEZ RUÍZ M, DÍEZ FÉREZ P, VIZCAÍNO LÓPEZ A, FERNÁNDEZ FERNÁNDEZ A, MARTÍNEZ PARDAVILA R, POU SANTONJA G, GRACIA MARZO A.: Adrenal Hemangioma: unusual cause of retroperitoneal hemorrhage. Servicio de Cirugía General, Hospital San Millán, Logroño, La Rioja, España. Arch Esp Urol 1995 jan-feb; 48 (1): 82-85.

Dra. M. Allué López

C/ Asalto, 67 - 5o D

50002 Zaragoza

(Trabajo recibido el 17 mayo de 2002) 